\title{
Liguzinediol improved the heart function and inhibited myocardial cell apoptosis in rats with heart failure
}

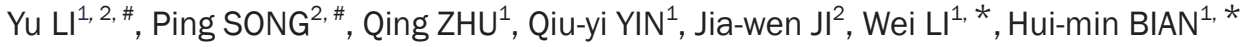 \\ ${ }^{1}$ Jiangsu Key Laboratory for Chinese Medicine Processing, Collage of Pharmacy, Nanjing University of Chinese Medicine, Nanjing \\ 210023, China; ${ }^{2}$ Department of Preclinical Medicine, Nanjing University of Chinese Medicine, Nanjing 210023, China
}

\begin{abstract}
Aim: Liguzinediol is a novel derivative of ligustrazine isolated from the traditional Chinese medicine Chuanxiong (Ligusticum wallichii Franch), and produces significant positive inotropic effect in isolated rat hearts. In this study we investigated the effects of liguzinediol on a rat model of heart failure.

Methods: To induce heart failure, male SD rats were injected with doxorubicin (DOX, $2 \mathrm{mg} / \mathrm{kg}$, ip) once a week for 4 weeks. Then the rats were administered with liguzinediol $\left(5,10\right.$, and $\left.20 \mathrm{mg} \cdot \mathrm{kg}^{-1} \cdot \mathrm{d}^{-1}, \mathrm{po}\right)$ for 2 weeks. Hemodynamic examination was conducted to evaluate heart function. Myocardial cell apoptosis was examined morphologically. The expression of related genes and proteins were analyzed using immunohistochemical staining and Western blot assays, respectively.

Results: Oral administration of liguzinediol dose-dependently improved the heart function in DOX-treated rats. Electron microscopy revealed that liguzinediol $\left(10 \mathrm{mg} \cdot \mathrm{kg}^{-1} \cdot \mathrm{d}^{-1}\right)$ markedly attenuated DOX-induced injury of cardiomyocytes, and decreased the number of apoptotic bodies in cardiomyocytes. Furthermore, liguzinediol significantly decreased Bax protein level, and increased Bcl-2 protein level in cardiomyocytes of DOX-treated rats, led to an increase in the ratio of Bcl-2/Bax. Moreover, liguzinediol significantly decreased the expression of both cleaved caspase-3 and NF-kB in cardiomyocytes of DOX-treated rats. Administration of digitalis $(0.0225$ $\mathrm{mg} \cdot \mathrm{kg}^{-1} \cdot \mathrm{d}^{-1}$ ) also markedly improved the heart function and the morphology of cardiomyocytes in DOX-treated rats.

Conclusion: Liguzinediol improves the heart function and inhibits myocardial cell apoptosis in the rat model of heart failure, which is associated with regulating $\mathrm{Bcl}-2$, Bax, caspase-3, and NF-kB expression.
\end{abstract}

Keywords: heart failure; cardiomyocyte; liguzinediol; apoptosis; caspases; Bcl-2; NF-kB; doxorubicin; Ligusticum wallichii

Acta Pharmacologica Sinica (2014) 35: 1257-1264; doi: 10.1038/aps.2014.75; published online 15 Sep 2014

\section{Introduction}

Liguzinediol (Figure 1) is a para-dihydroxy derivative of ligustrazine [4-methyl-pyrazine (tetramethylpyrazine), $\mathrm{TMP}]^{[1]}$, which was isolated from the traditional Chinese medicine herb Chuanxiong (Ligusticum wallichii Franch) and used for the treatment of angina pectoris. Ligustrazine plays a role

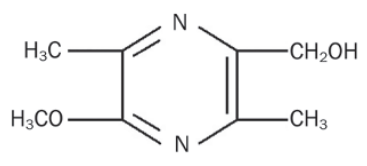

Figure 1. Structure of liguzinediol.

\footnotetext{
\# These authors contributed equally to this manuscript.

* To whom correspondence should be addressed.

E-mail hmbian@sina.com (Hui-min BIAN); liwaii@126.com (Wei LI)

Received 2014-03-05 Accepted 2014-07-06
}

in expanding blood vessels, increasing both coronary and cerebral blood flow, preventing platelet aggregation, inhibiting thrombosis, and improving microcirculation ${ }^{[2]}$. It also decreases hematological indices of myocardial ischemia in rats and protects against acute myocardial ischemia and myocardial ischemia-reperfusion injury ${ }^{[3]}$. Through the study of TMP derivatives, we unexpectedly found that liguzinediol (which has been patented ${ }^{[4]}$ ) has significant positive inotropic effects, which were stronger than those of TMP. Additionally, it does not cause arrhythmia, hypotension or other side effects. Early experiments have found that liguzinediol has unique biological properties that may prove effective in treating heart failure $^{[5]}$.

Cardiovascular diseases, particularly heart failure (HF), are the most common causes of human death worldwide ${ }^{[6]}$. In China's rapidly aging population, HF has become a serious public health problem with an estimated 4.2 million patients experiencing HF in $2010^{[7]}$. The British Heart Foundation (BHF) estimates that around 750000 people in the UK have HF 
and there are approximately 25000 new cases each year. Prognosis from $\mathrm{HF}$ is poor and the 5-year survival rate for patients with $\mathrm{HF}$ is estimated to be only $58 \%^{[8-10]}$. Myocardial cell apoptosis has become an increasingly important concept in the pathogenesis of heart failure. Cardiac myocyte apoptosis is an important factor in heart failure. Apoptosis is observed in cardiac myocytes in failing hearts, and has been implicated in the progression of cardiomyopathy ${ }^{[10]}$. We hypothesized that liguzinediol may protect cardiomyocytes from apoptosis induced by doxorubicin (DOX) and subsequently improve heart function. To test this hypothesis, we performed experiments in a rat model of DOX-induced apoptosis and explored the mechanisms underlying the results of these experiments.

\section{Materials and methods Materials}

Liguzinediol (purity $>99 \%$ ) was provided by Prof Wei LI (Nanjing University of Chinese Medicine). Doxorubicin hydrochloride was purchased from the Beijing Huafeng United Corporation. All antibodies used in the present study were obtained from Santa Cruz Technology (Santa Cruz, CA, USA). All other reagents and kits were from commercial suppliers and were of the standard biochemical quality.

\section{Rat DOX-induced heart failure model}

The animal experimental protocols were approved by the Nanjing University of Chinese Medicine Committee on Laboratory Animal Care, and all animals received humane care according to the National Institutes of Health (USA) guidelines. SpragueDawley rats were obtained from the Shanghai Laboratory Animal Center. All animals were housed under diurnal lighting conditions and allowed food and water ad libitum. The animal model was established based on the methods reported by Sun $e t a^{[11]}$, and a modified animal model of doxorubicininduced cardiotoxicity was utilized for the study. Forty-eight male Sprague-Dawley rats weighing $250 \pm 20 \mathrm{~g}$ were randomly divided into a control group (normal saline, NS, $n=8$ ) and a DOX group $(2 \mathrm{mg} / \mathrm{kg})$. NS and DOX $(2 \mathrm{mg} / \mathrm{kg})$ were administered via intraperitoneal injections once per week to establish a chronic heart failure model. After 4 weeks, DOX-treated rats were divided into 5 groups according to their left ventricular systolic pressures (LVSP): these groups included a DOX group, a $5 \mathrm{mg} / \mathrm{kg}$ liguzinediol group, a $10 \mathrm{mg} / \mathrm{kg}$ group liguzinediol group, a $20 \mathrm{mg} / \mathrm{kg}$ liguzinediol group, and a $0.0225 \mathrm{mg} / \mathrm{kg}$ digitalis group. NS and liguzinediol were each administered gastrically once per day. Hemodynamic examination was conducted using a 4-channel Biological System. Rat hearts were analyzed via electron microscopy, Western blot analyses, and immunohistochemistry after 2 weeks.

\section{Hemodynamic examination}

Hemodynamic examinations were performed as previously described $^{[12]}$. A $0.7 \mathrm{~cm}$ neck incision was made to expose the right common carotid artery, and a catheter was inserted approximately $1.5 \mathrm{~cm}$ from the heart. Arterial blood pressure was monitored for $30 \mathrm{~min}$, and the catheter was subsequently inserted into the ventricular chamber to record intraventricular pressures for $30 \mathrm{~min}$. Changes in hemodynamic parameters, including the heart rate (HR), left ventricular systolic pressure (LVSP), the maximal rate of rise in blood pressure of the ventricular chamber $\left(+\mathrm{d} p / \mathrm{d} t_{\max }\right)$ and the maximal rate of decline in blood pressure of the ventricular chamber $\left(-\mathrm{d} p / \mathrm{d} t_{\max }\right)$ were measured and recorded using a 4-channel Biological System (RM-6240, Chengdu Instrument Factory, Chengdu, China).

\section{HE staining}

Following anesthesia, the rat hearts were excised and immediately placed in $10 \%$ neutral-buffered formalin, wherein they were kept at room temperature for $24 \mathrm{~h}$. The heart specimens were embedded in paraffin, cut into 5 - $\mu \mathrm{m}$ sections, stained with hematoxylin for $5 \mathrm{~min}$, and stained with eosin for another $5 \mathrm{~min}$, at room temperature. After three more $5 \mathrm{~min}$ washes in PBS, sections were quickly dedydrated in 95\% alcohol, made transparent in dimethylbenzene, and sealed using neutral resin. Finally, the percentage of damaged cells was counted in 100 sections per animal for all 48 animals and averaged for further analysis.

\section{Electron microscopic examination of ultrastructural alterations in rat hearts}

An ultrastructural analysis of heart morphological changes was performed to search for evidence of apoptosis in the manner described by Peter et al ${ }^{[13]}$. Hearts were immersed in $4 \%$ buffered paraformaldehyde at autopsy and grossly sectioned before undergoing further fixation. Hearts were separated into $1 \mathrm{~cm} \times 0.5 \mathrm{~cm} \times 0.5 \mathrm{~cm}$ sections, and each section was repeatedly osmicated in $1 \%$ osmium tetroxide. Following dehydration with ethanol, the samples were embedded in epoxy resin. Ultrathin sections were then mounted on nickel grids and stained with uranyl acetate and lead citrate. A Philip CM 120 electron microscope (FEI Inc, Eindhoven, Netherlands) was used for subsequent analyses.

\section{Western blot analysis of proteins in left ventricular tissues}

The frozen hearts were cut into small pieces and homogenized in $0.5 \mathrm{~mL}$ of RIPA buffer $(150 \mathrm{mmol} / \mathrm{L} \mathrm{NaCl}, 1 \%$ Nonidet $\mathrm{P} 40,0.5 \%$ deoxycholate, $0.1 \% \mathrm{SDS}, 50 \mathrm{mmol} / \mathrm{L}$ Tris/ $\mathrm{HCl}$ and $2 \mathrm{mmol} / \mathrm{L}$ PMSF, $\mathrm{pH}$ 7.4) before being transferred into small tubes and rotated at $4{ }^{\circ} \mathrm{C}$ overnight. Solubilized proteins were collected following centrifugation at $10000 \times g$ for $30 \mathrm{~min}$. The supernatant was collected and stored at $80^{\circ} \mathrm{C}$. Whole cell proteins were used to test the expression of apoptotic genes, including Bax, Bcl-2, and caspase-3. The protein concentrations of each sample were quantified using an enhanced BCA (bicinchoninic acid) Protein Assay kit (Beyotime Biotechnology, Haimen, China). Protein lysates from each group of rats were separated using SDS/PAGE and electrotransferred onto a polyvinylidene fluoride (PVDF) membrane. Non-specific proteins were blocked with $5 \%$ non-fat dried skimmed milk powder prepared in TBS $+0.1 \%$ Tween 20 for $2 \mathrm{~h}$ at room temperature. Immunoblotting was then performed using 2 $\mu \mathrm{g} / \mathrm{mL}$ rabbit anti-rat procaspase-3 polyclonal antibody (Santa 
Cruz, USA), rabbit anti-human cleaved caspase-3 polyclonal antibody (Santa Cruz, USA), rabbit anti-human Bax polyclonal antibody (Santa Cruz, USA), and rabbit anti-human Bcl-2 polyclonal antibody (Santa Cruz, USA). Membrane blots were washed and incubated with either horseradish-peroxidaseconjugated anti-rabbit IgG antibodies or anti-mouse IgG antibodies. Immunoreactive proteins were subsequently visualized using ECL ${ }^{\circledR}$ plus, a Western blot detection system (R\&D Systems).

\section{Histological examination and immunohistochemical staining}

Rat hearts were immediately fixed in formalin. Following dehydration with xylene, the samples were embedded in wax, and 5-mm sections were made. Prior to sectioning, samples from hearts were stained with hematoxylin and eosin. For immunohistochemical staining, sections were dewaxed and microwaved for $5 \mathrm{~min}$ at $500 \mathrm{~W}$ with antigen retrieval solution (10 mmol/L sodium citrate, $\mathrm{pH} 6)$. The sections were allowed to cool in $0.1 \mathrm{~mol} / \mathrm{L}$ Tris- $\mathrm{HCl}, \mathrm{pH} 8$, prior to reduction with $10 \mathrm{mmol} / \mathrm{L}$ dithiotreitol (DTT) and alkylation with $25 \mathrm{mmol} / \mathrm{L}$ iodoacetamide: each treatment was applied for $30 \mathrm{~min}$. Endogenous peroxidase activity was quenched for 30 min using 3\% $(v / v)$ hydrogen peroxide in methanol at room temperature. Tissue sections were blocked with $10 \%$ $(v / v)$ donkey serum and $1 \%(w / v)$ bovine serum albumin in PBS (blocking solution) for $1 \mathrm{~h}$ prior to their incubation with protein A-purified mucin-specific antibodies (diluted 1:100 in the blocking solution for $1 \mathrm{~h}$ at room temperature). Sections were then incubated for $30 \mathrm{~min}$ with biotin labeled goat antirabbit IgG (Santa Cruz, Santa Cruz, USA), diluted 1:250 in the blocking solution, and then treated with streptavidin (ABC Kit Elite standard, VectaStain) for $30 \mathrm{~min}$ prior to detection with Diaminobenzidine (Sigma, St Louis, USA). All sections were counterstained with Harris's hematoxylin, and representative pictures were taken using an Axiovision microscope. The integral optical density (IOD) value of the immunohistochemical picture was analyzed via Imagepro-Plus software.

\section{Statistical analysis}

Data are expressed as the mean \pm SEM based on three to six independent experiments. The paired Student's $t$-test was used to evaluate statistical significance. A $P$ value of less than
0.05 was considered statistically significant.

\section{Results}

Liguzinediol improves heart function in DOX-treated rats

Hemodynamic indices were recorded following 2 weeks of liguzinediol administration. As shown in Table 1, liguzinediol significantly improved LVSP, LV $+\mathrm{d} p / \mathrm{d} t_{\max }$, and $\mathrm{LV}-\mathrm{d} p / \mathrm{d} t_{\max }$, and also caused an increase in HR, compared with the DOX group. These observations suggest that liguzinediol improved the heart function of rats with DOX-induced injury.

\section{Liguzinediol improves morphological alterations of myocardial fibers in DOX-treated rats}

In the control group, myocardial fibers were properly organized, and their structures were normal. There were no broken fibers or inflammatory cells. The nuclei of these myocardial cells were normal. In the DOX group, myocardial fibers appeared broken and thickened, and these fiber abnormalities were accompanied by inflammatory cell infiltration. However, liguzinediol administration limited the number of myocardial fiber fractures and decreased the extent of inflammatory cell infiltration, an effect that correlated positively with the dose administered. Moreover, digitalis also significantly reduced myocardial fiber damage, as these myocardial fibers had an almost normal appearance despite a small amount of lipofuscin deposition (Figure 2).

\section{Liguzinediol attenuates ultrastructural alterations in cardio- myocytes}

Ultrastructural modifications were observed in liguzinedioltreated rats. In the control group, the nuclei were big, round, or oval, and characterized by homogeneously distributed chromatin, and the mitochondria were characterized by tightly packed, intact, horizontally oriented cristae. However, after exposure to DOX, the nucleolemma disappeared, and nucleic condensation was observed. The mitochondrial cristae also disappeared, and mitochondrial vacuolization and swelling were observed. By contrast, after liguzinediol treatment, the majority of cardiomyocytes demonstrated ultrastructural profiles that appeared virtually unaltered, as they were characterized by normal appearing nuclei, mitochondria with only minimal swelling, and normal appearing lamellar cristae. The

Table 1. Effects of liguzinediol on hemodynamic parameters and cardiac function in Dox-treated rats in vivo. Data are expressed as mean $\pm S E M$. $n=8$ in each group. ${ }^{\mathrm{c}} P<0.01$ vs control. ${ }^{\mathrm{e}} \mathrm{P}<0.05,{ }^{\mathrm{f}} \mathrm{P}<0.01$ vs DOX.

\begin{tabular}{|c|c|c|c|c|c|c|}
\hline & Control & Dox & L 5 mg/kg & L 10 mg/kg & L 20 mg/kg & Dig $0.0225 \mathrm{mg} / \mathrm{kg}$ \\
\hline SBP (mmHg) & $99.84 \pm 15.92$ & $73.07 \pm 17.55^{c}$ & $77.8 \pm 7.10$ & $95.04 \pm 13.01^{\mathrm{e}}$ & $90.37 \pm 13.28^{\mathrm{e}}$ & $82.42 \pm 18.75$ \\
\hline $\mathrm{DBP}(\mathrm{mmHg})$ & $58.11 \pm 19.22$ & $51.85 \pm 17.97$ & $56.09 \pm 8.87$ & $75.36 \pm 13.04^{f}$ & $73.74 \pm 11.16^{\mathrm{e}}$ & $62.18 \pm 20.38$ \\
\hline$+\mathrm{LV} \mathrm{d} p / \mathrm{d} t_{\max }(\mathrm{mmHg} / \mathrm{s})$ & $7158.67 \pm 1787.06$ & $3256.35 \pm 1594.88^{c}$ & $5004.56 \pm 1874.28$ & $5693.05 \pm 558.32^{f}$ & $6502.72 \pm 1182.04^{f}$ & $6270.55 \pm 937.01^{f}$ \\
\hline$-\mathrm{LV} \mathrm{d} p / \mathrm{d} t_{\max }(\mathrm{mmHg} / \mathrm{s})$ & $5731.81 \pm 1541.35$ & $2956.25 \pm 1396.04^{c}$ & $4212.46 \pm 797.81^{b}$ & $4845.39 \pm 764.72^{f}$ & $5548.26 \pm 1289.85^{f}$ & $4984.65 \pm 1060.00^{f}$ \\
\hline
\end{tabular}

SBP, systolic blood pressure; DBP, diastolic blood pressure; HR, heart rate; LVSP, left ventricular systolic pressure; $\pm \mathrm{LV} d p / d t_{\max }$, the maximal rates of increase and decrease of left-ventricle pressure development. 

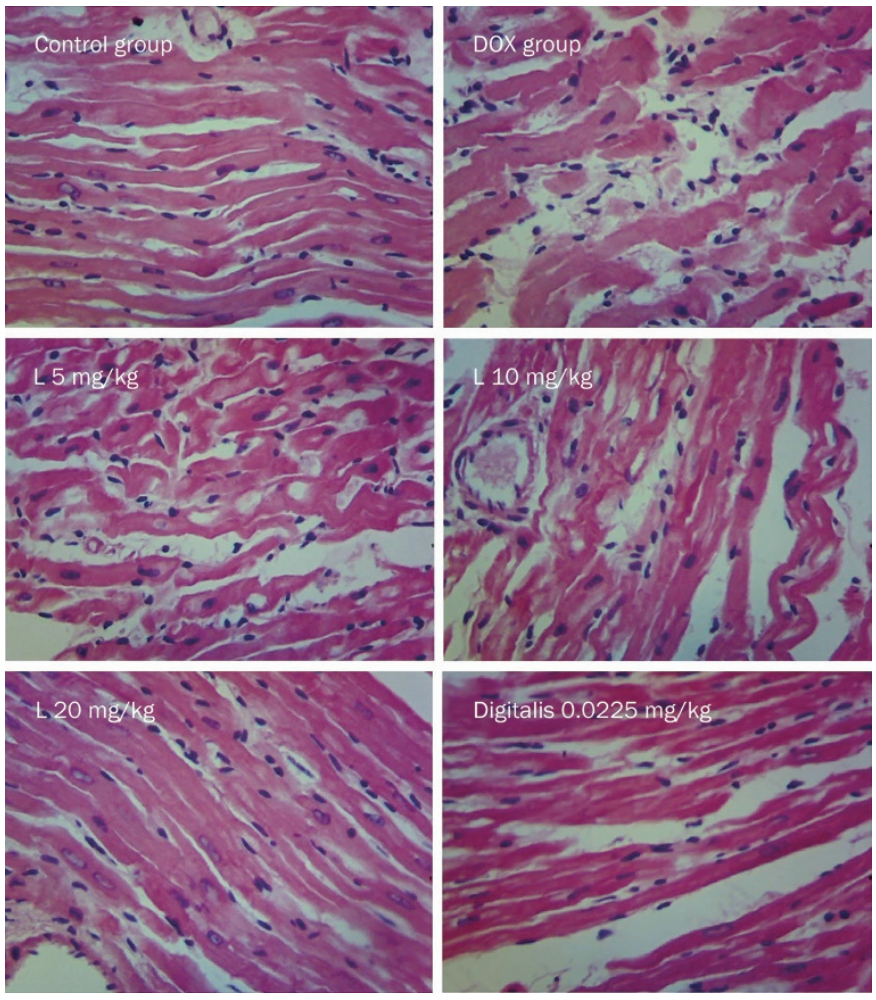

Figure 2. Liguzinediol improves DOX-induced morphologic alterations in cardiomyocytes examined by HE staining $(\times 400)$.

incidence of apoptosis was decreased in the digitalis group (Figure 3).
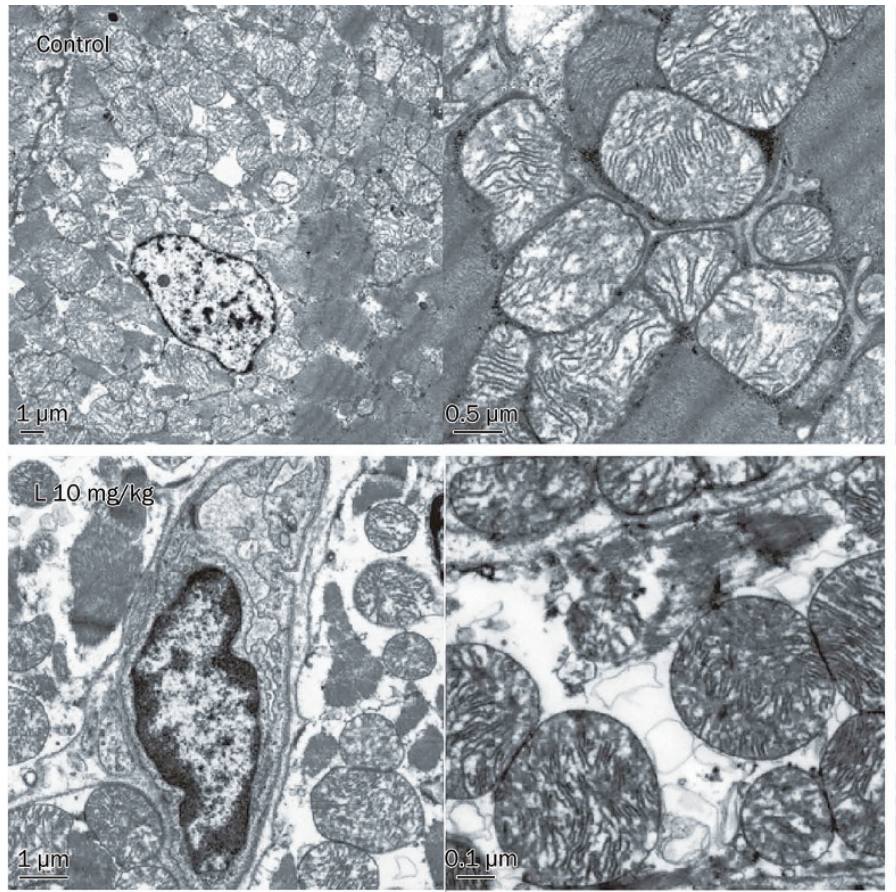

Liguzinediol reduces the ratio of $\mathrm{Bax} / \mathrm{Bcl}-2$ in cardiomyocytes

As illustrated in Figure 4, a Western blot assay was utilized to detect protein expression levels of Bax and Bcl-2. The Bcl-2 protein content of the $10 \mathrm{mg} / \mathrm{kg}$ liguzinediol group $(0.71 \pm 0.06)$ was higher than that of the DOX group $(0.54 \pm 0.01)$, a difference with a statistical significance of $P<0.01$ (Figure 4A). The Bax protein content of the $10 \mathrm{mg} / \mathrm{kg}$ liguzinediol group $(1.07 \pm 0.08)$ was significantly lower than that of the DOX group $(1.48 \pm 0.03, P<0.01)$ (Figure 4B). The ratio of Bax/Bcl-2 in the $10 \mathrm{mg} / \mathrm{kg}$ liguzinediol group (1.52 \pm 0.06$)$ was significantly lower than that of the DOX group $(2.74 \pm 0.08, P<0.01)$ (Figure $4 \mathrm{C})$. These results suggest that liguzinediol decreases cardiomyocyte apoptosis through the up-regulation of Bcl-2 and the down-regulation of Bax. Bcl-2 and Bax immunostaining confirmed the above results (Figure 5).

\section{Liguzinediol inhibits caspase-3 in cardiomyocytes}

A Western blot assay was utilized to detect the protein levels of both pro-caspase-3 and cleaved-caspase-3. The procaspase- 3 protein content of the $10 \mathrm{mg} / \mathrm{kg}$ liguzinediol group $(0.94 \pm 0.05)$ was higher than that of the DOX group $(0.75 \pm 0.04)$, a difference with a statistical significance of $P<0.01$. The cleaved-caspase- 3 protein content of the $10 \mathrm{mg} / \mathrm{kg}$ liguzinediol group (1.85 \pm 0.09$)$ was significantly lower than that of the DOX group $(2.59 \pm 0.29, P<0.05)$ (Figure 6). These results suggest that liguzinediol decreases cardiomyocyte apoptosis via the inhibition of caspase-3.

\section{Liguzinediol reduces cardiomyocyte apoptosis via the inhibition of NF-KB}

Our results showed that the immunostaining of NF-KB was

Figure 3. Liguzinediol suppresses DOX-induced morphologic alterations in cardiomyocytes examined by an electron microscope. Red arrows indicate apoptotic bodies, and blue arrows indicate mitochondrial ultrastructures.
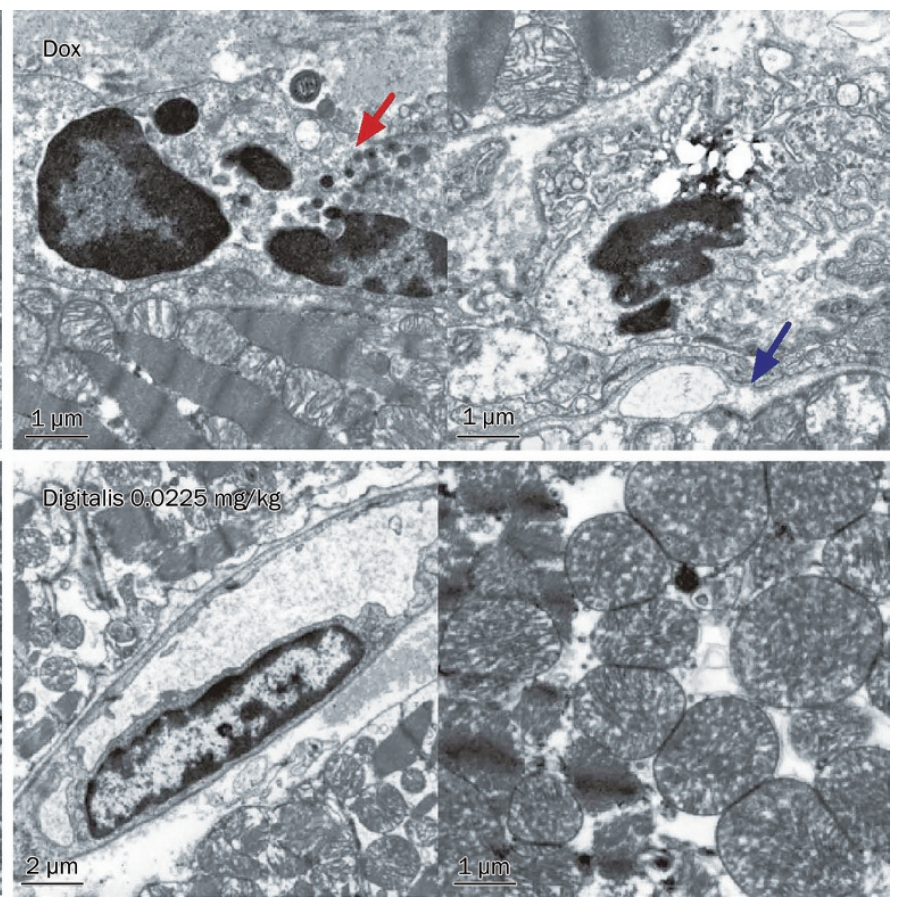
A

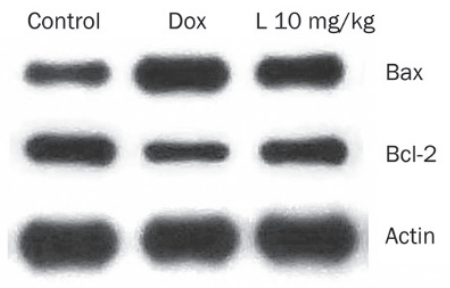

B

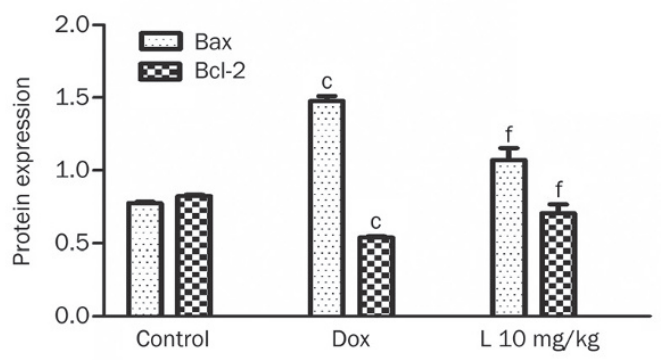

C

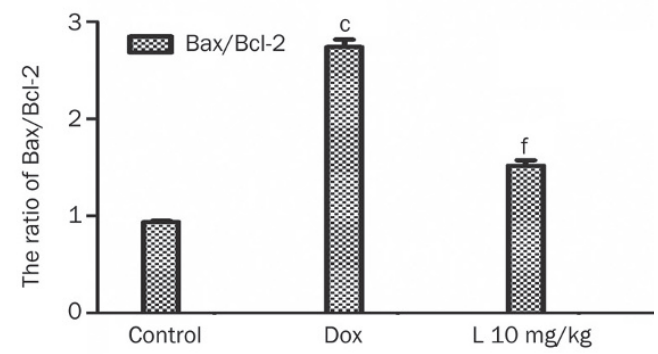

Figure 4. Liguzinediol decreases the ratio of $\mathrm{Bax} / \mathrm{Bcl}-2$ in cardiomyocytes. (A) Bax and $\mathrm{Bcl}-2$ protein expression as determined by Western blot assays. (B) SDS/PAGE analysis shows changes in Bax and $\mathrm{Bcl}-2$ protein expression. ${ }^{\mathrm{b}} P<0.05,{ }^{\mathrm{C}} P<0.01$ vs the control group. ${ }^{\mathrm{e}} P<0.05,{ }^{\mathrm{f}} P<0.01$ vs the DOX group $(n=3)$. (C) SDS/PAGE analysis shows the ratio of Bax to Bcl-2 protein expression. ${ }^{b} P<0.05,{ }^{c} P<0.01$ vs the control group. ${ }^{\mathrm{e}} P<0.05$, ${ }^{f} P<0.01$ vs the DOX group $(n=3)$.

weak and was characterized by the appearance of a small amount of light brown staining but became stronger in the DOX group, as much larger amounts of positive dark brown immunostaining appeared. These data indicated that the expression level of activated NF-KB was increased in DOX group. In the $10 \mathrm{mg} / \mathrm{kg}$ liguzinediol group, the staining of NF- $\mathrm{KB}$ was weaker than in the DOX group, suggesting that liguzinediol attenuates the activation of NF-kB (Figure 7).

\section{Discussion}

Hemodynamics are important indicators of cardiac function. Hemodynamic changes are key indicators of both the systolic and the diastolic function of the heart. Among these indicators are LVSP and $+\mathrm{d} p / \mathrm{dt}_{\max }$, which reflect myocardial contractile function. Left ventricle end diastolic pressure (LVEDP) and $-\mathrm{d} p / \mathrm{d} t_{\max }$ reflect myocardial relaxation ${ }^{[14]}$. Our hemodynamic examinations revealed that liguzinediol and digitalis significantly increased LVSP, $+\mathrm{d} p / \mathrm{d} t_{\max }, \mathrm{SBP}, \mathrm{DBP}$, and HR, but decreased $-\mathrm{d} p / \mathrm{d} t_{\max }$, compared with the control group. The effects of liguzinediol at 10 and $20 \mathrm{mg} / \mathrm{kg}$ and digitalis at $0.0225 \mathrm{mg} / \mathrm{kg}$ on LVSP, $+\mathrm{d} p / \mathrm{d} t_{\max }$ and $-\mathrm{d} p / \mathrm{d} t_{\max }$ were stronger than the effects exerted by liguzinediol at $5 \mathrm{mg} / \mathrm{kg}$, indicating that liguzinediol has positive inotropic effects and mitigates the changes associated with chronic heart failure induced by Dox.

The pathological changes noted in myocardial tissue have a unique diagnostic significance for heart failure, as they are visible and objectively reflect the pathological damage inflicted on myocardial cells. Our present study showed that the arrangement of myocardial cells in Dox-treated rats was disordered, and the interstitial tissue became fibrotic. The myocardial fibers were fractured, and the myocardial vacuoles were degenerative. Inflammatory cells infiltrated areas around the myocardial cells. However, each of these pathological alterations was mitigated by liguzinediol, indicating that liguzinediol exerts protective effects on myocardial cells.

Myocardial cell ultrastructures were observed using an electron microscope. The results of these observations confirmed the morphological features associated with myocardial cell apoptosis. The myocardial cells of the Dox group demonstrated larger amounts of poorly concentrated heterochromatin, as well as the existence of apoptotic bodies. By contrast, we observed larger amounts of euchromatin in liguzinedioltreated rats, but there were no apoptotic bodies. There were fewer swollen mitochondria, and the lamellar cristae within the mitochondria appeared normal.

It has been reported that numerous signaling molecules, such as cytochrome $c, \mathrm{Bcl}-2, \mathrm{Bax}, \mathrm{p} 53$, and Fas, have been implicated in the apoptotic pathways of cardiomyocytes ${ }^{[15,16]}$. Bcl-2 family members include Bax, Bcl-2, and Bcl-xl. Bcl-2 was the first cell survival factor discovered in mammals, as it functions as an anti-apoptotic gene and is a key factor in regulating both physiological and pathological apoptosis. When the ratio of Bcl-2/Bax is increased, the dimer facilitates cell survival; however, when the ratio is decreased, the dimer facilitates apoptosis ${ }^{[17,18]}$. The present study showed no expression of the Bcl-2 protein, but did show significant expression of the Bax protein in the DOX group, as well as the existence of minor $\mathrm{Bcl}-2$ proteins in light color and abundant Bax proteins in dark color, indicating a close association between the expression of Bcl-2 and Bax proteins and apoptosis. The expression of the Bax and Bcl-2 proteins in cardiac muscle cells treated with liguzinediol was affected much more significantly than the expression of these proteins in the DOX group, suggesting that liguzinediol inhibits the apoptotic process by up-regulating Bcl-2 expression and down-regulating Bax expression. By gradually increasing the ratio of $\mathrm{Bcl}-2 / \mathrm{Bax}$, liguzinediol played a role in the regulation of cardiomyocyte apoptosis.

The caspase family is a key player in apoptosis. Our studies confirmed the involvement of caspase proteins in liguzinediol's regulation of cardiomyocyte apoptosis. It is generally known that caspase-3 plays a pivotal role in mammalian apoptotic pathways. Several apoptosis-stimulating factors trigger the activation of different proteases, which leads to the activation of the caspase- 3 proenzyme and the subsequent activation of caspase-3. Activated caspase-3 subsequently triggers prote- 
A
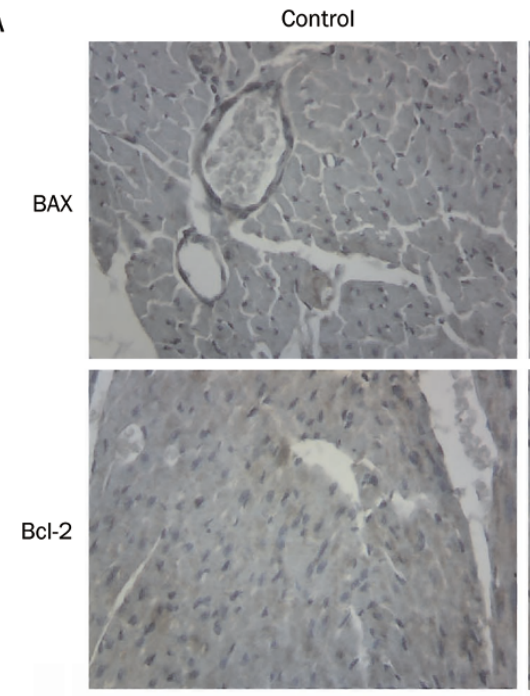
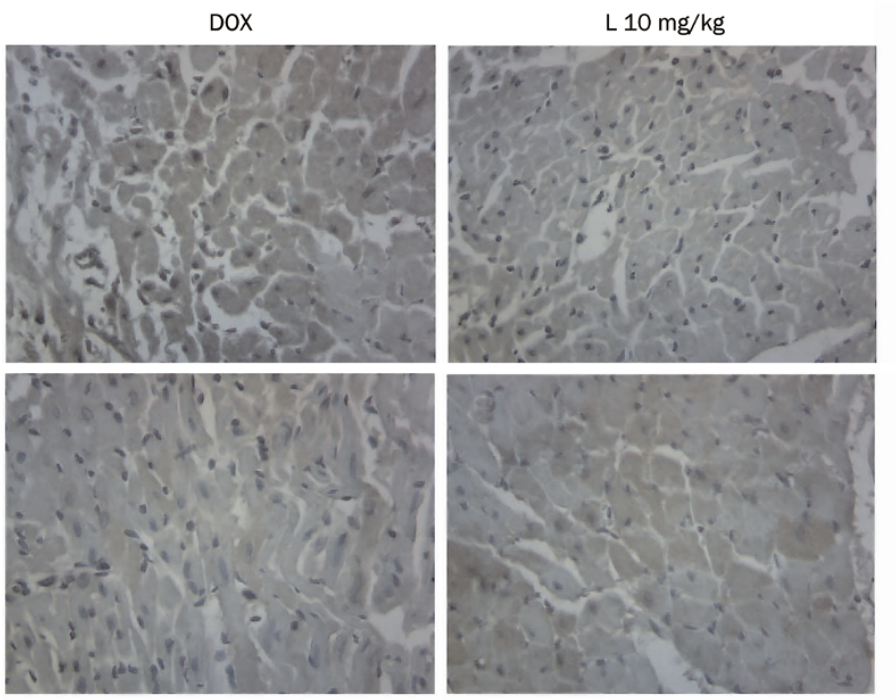

B

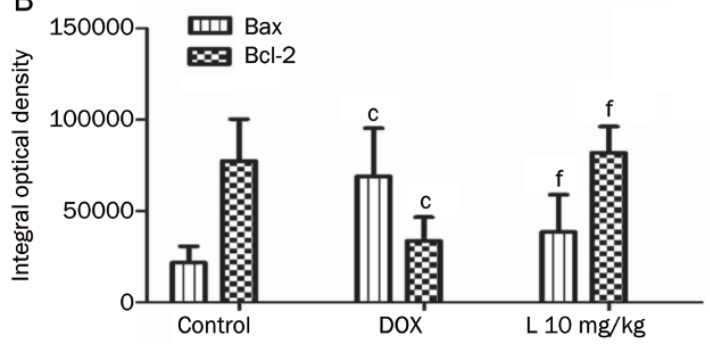

C

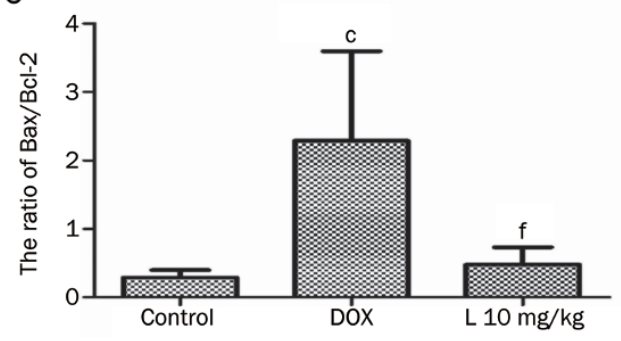

Figure 5. Bcl-2 and Bax immunoreactivity in cardiomyocytes. The upper two panels show the basal expression levels of Bcl-2 and Bax in the control group. The immunoreactivity of Bax in the DOX group was higher than in the $10 \mathrm{mg} / \mathrm{kg} \mathrm{L}$ group. The immunoreactivity of Bcl-2 in the DOX group (red arrows) was lower than in the $10 \mathrm{mg} / \mathrm{kg} \mathrm{L}$ group. ${ }^{\mathrm{c}} P<0.01 \mathrm{vs}$ the control group. ${ }^{\mathrm{e}} P<0.05,{ }^{\mathrm{f}} P<0.01 \mathrm{vs}$ the DOX group ( $\left.n=8\right)$.

A

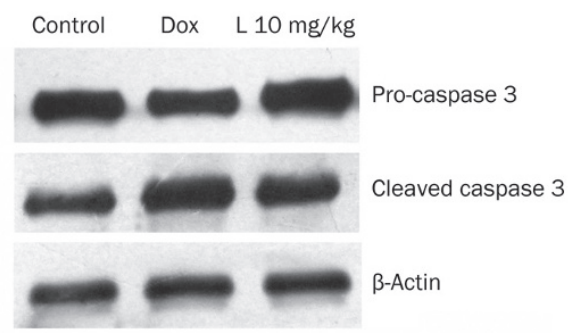

B

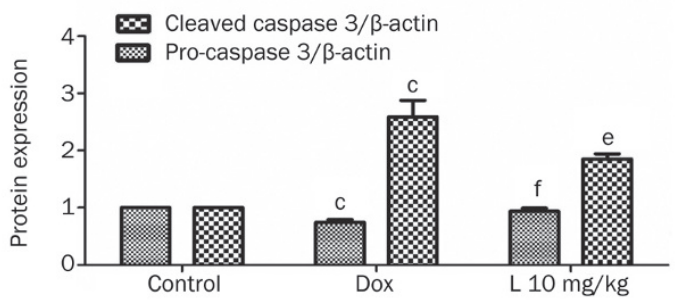

Figure 6. The expression of pro-caspase-3 and cleaved caspase-3 using Western blot assays. (A) Pro-caspase-3 and cleaved caspase-3 protein expression as determined by Western blot assays. (B) SDS/PAGE analysis demonstrates changes in cleaved caspase-3 and pro-caspase-3 protein expression levels. ${ }^{\mathrm{b}} P<0.05,{ }^{\mathrm{c}} P<0.01$ vs the control group. ${ }^{\mathrm{e}} P<0.05$, ${ }^{\mathrm{f}} P<0.01$ vs the DOX group $(n=3)$. ases to cleave corresponding substrates ${ }^{[19]}$. Our studies demonstrated a more significant increase in the level of activated caspase-3 in DOX-treated cardiomyocytes than in normal cardiomyocytes, findings that confirmed the relationship between caspase- 3 and cardiomyocyte apoptosis. Given the decreased caspase-3 levels noted in liguzinediol-treated cardiomyocytes, it is assumed that liguzinediol inhibits apoptosis by blocking caspase activation cascades.

NF-kB is reportedly involved in regulating DOX-induced apoptosis in various cancer cells and carcinomas, including breast-cancer cells, pancreatic carcinoma cells, leukemia, lymphoma, renal carcinoma and hepatocellular carcinoma ${ }^{[20-22]}$. DOX-induced NF-kB activation in tumor cells has antiapoptotic effects. Inhibition of NF-KB activation leaves cancer cells vulnerable to DOX-induced apoptosis ${ }^{[20-22]}$. Suwei $e t$ $a l^{[23]}$ provided the first known evidence that NF-kB activation promotes DOX-induced apoptosis in myocytes. The proapoptotic character of NF-KB may be due to its direct activation of apoptotic genes, including Fas ligand, Fas, c-Myc, and $\mathrm{p} 53^{[24-26]}$. It has also been proposed that NF-kB down-regulates the activities of some anti-apoptotic factors, such as $\mathrm{Bcl}-\mathrm{X}_{\mathrm{L}}{ }^{[27]}$. Additionally, the involvement of NF-KB in regulating the cell cycle may also be a mechanism by which it exerts its proapop- 

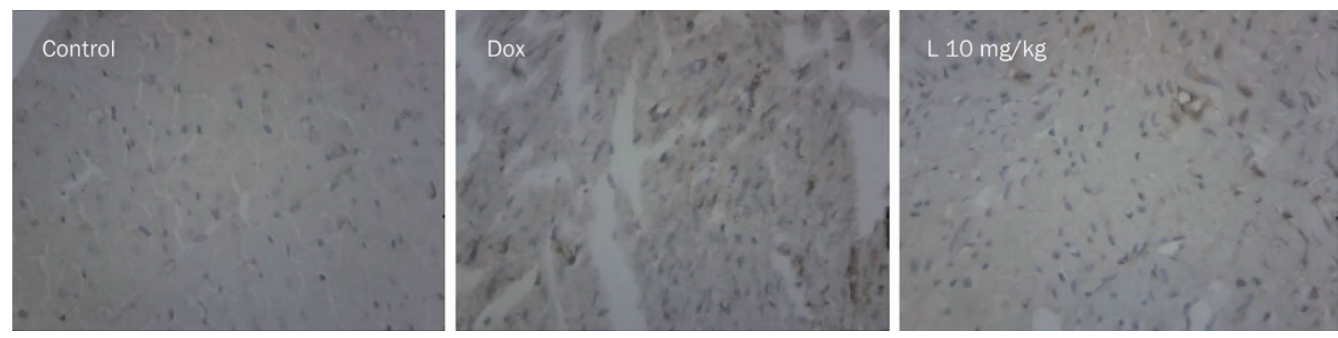

Figure 7. NF-kB immunoreactivity in cardiomyocytes. The left panel shows the basal expression level of NF-kB in the control group. The NF-kB expression level was increased in the Dox group and was higher than in the control group. Treatment with liguzinediol at a dose of $10 \mathrm{mg} / \mathrm{kg}$ decreased the level of NF-KB expression $(n=8)$.

totic effects ${ }^{[26]}$. In the present study, we demonstrated that doxorubicin-induced activation of NF-kB leads to myocardial apoptosis. However, $10 \mathrm{mg} / \mathrm{kg}$ of liguzinediol decreased NF-kB expression compared with the DOX group. Therefore, liguzinediol may attenuate DOX-induced activation of NF-kB.

The experimental results showed that the number of apoptotic myocardial cells was significantly increased in rats with a DOX-induced heart failure, but this could not be seen in control rats. Treatment with liguzinediol and digoxin significantly reduced the apoptotic myocardial cells compared with the model rats. This result indicated that myocardial cell apoptosis was tightly associated with the development of heart failure and that both liguzinediol and digoxin can inhibit myocardial cell apoptosis in rats. Myocardial cell apoptosis represents one of the most key mechanisms underlying heart failure. Decreased number of myocardial cells will exacerbate the process of fibrosis eventually leading to heat failure.

In summary, our data have demonstrated the efficacy of liguzinediol in reducing apoptosis in myocardial cells in rats with heart failure. Mechanistic studies showed that liguzinediol inhibits cardiomyocyte apoptosis by regulating the balance between Bax and Bcl-2, blocking caspase cascades, and inhibiting NF-kB activation.

\section{Acknowledgements}

This study is supported by the National Natural Science Foundation of China (№ 81072542), the Natural Science Foundation of Jiangsu Province (No BK2011077), and the Research Fund for the Doctoral Program of Higher Education (No 20123237110010), as well as the College Student's Innovative Training Program, located in Jiangsu Province.

\section{Author contribution}

Wei LI and Hui-min BIAN designed the research. Yu LI, Qing ZHU, and Qiu-yi YIN performed the experiments. Ping SONG and Jia-wen JI analyzed the data, and Yu LI wrote the paper.

\section{References}

1 Liu Z, Li W, Wen HM, Bian HM, Zhang J, Chen L, et al. Synthesis, biological evaluation, and pharmacokinetic study of novel liguzinediol prodrugs. Molecules 2013; 18: 4561-72.

2 Dou $\mathrm{YZ}$, Teng $\mathrm{H}$, Wang $\mathrm{Q}$. Preparation, in vitro release and percutaneous penetration of ligustrazine hydrochloride transdermal delivery system. Chin J Pharm 2008; 39: 745-9.

3 Liu X, Liu H, Zeng Z, Zhou W, Liu J, He Z. Pharmacokinetics of ligustrazine ethosome patch in rats and anti-myocardial ischemia and anti-ischemic reperfusion injury effect. Int J Nanomed 2011; 6: 1391-8.

4 Li W, Chen L, Bian HM. Application of 2,5-dihydroxymethyl-3,6dimethylphrazine and its derivates in pharmacy. U S Patent 8, 158, 630, 17 April 2012.

5 Chen L, Xu Y, Li W, Wu H, Luo Z, Li X, et al. The novel compound liguzinediol exerts positive inotropic effects in isolated rat heart via sarcoplasmic reticulum $\mathrm{Ca}^{2+}$ ATPase-dependent mechanism. Life Sci 2012; 91: 402-8.

6 Lloyd-Jones D, Adams R, Carnethon M, De SG, Ferguson TB, Flegal $\mathrm{K}$, et al. Heart disease and stroke statistics-2009 update: a report from the American Heart Association Statistics Committee and Stroke Statistics Subcommittee. Circulation 2009; 119: 480-6.

7 Hu SS, Kong LZ, Gao RL, Zhu ML, Wang W, Wang YJ, et al. Outline of the report on cardiovascular disease in China, 2010. Biomed Environ Sci 2012; 25: 251-6.

8 British Heart Foundation. Coronary heart disease statistics. BHF, 2012. http://www.heartstat s.org

9 British Heart Foundation. Heart health conditions: heart failure. BHF, 2013. http://www.bhf.org.uk

10 National Clinical Guideline Centre (UK). Chronic Heart Failure- National clinical guideline for diagnosis and management in primary and secondary care. Royal College of Physicians (UK); 2010.

11 Sun JB, Yu CN, Xu D. Ginseng aconite and its small complex prescription shenfu decoction inhibiting doxorubicin-induced cardiotoxicity in rats. Liaoning J Tradit Chin Med 2012; 4: 754-6.

12 Yuan Y, Wang X, Zeng Q, Wu HM, Qi YF, Tang CS. Effects of continuous intermedin infusion on blood pressure and hemodynamic function in spontaneously hypertensive rats. J Geriatr Cardiol 2012; 9: 17-27.

13 Working PK, Newman MS, Sullivan T, Yarrington J. Reduction of the cardiotoxicity of doxorubicin in rabbits and dogs by encapsulation in long-circulating, pegylated liposomes. J Pharmacol Exp Ther 2012; 289: 1128-33.

14 Lai Y, Wu HJ, Zheng SD, Wu DL. The hemodynamic effects on chronic heart failure rats with Shen Fu Tang. Jilin J Tradit Chin Med 2013; 33: 276-77.

15 Nakamura T, Ueda Y, Juan Y, Katsuda S, Takahashi H, Koh E. Fasmediated apoptosis in adriamycin-induced cardiomyopathy in rats in vivo study. Circulation 2000; 102: 572-8.

16 Abd El-Gawad HM, El-Sawalhi MM. Nitric oxide and oxidative stress in brain and heart of normal rats treated with doxorubicin: role of aminoguanidine. J Biochem Mol Toxicol 2004; 18: 69-77.

17 Perez GI, Robles R, Knudson CM, Flaws JA, Korsmeyer SJ, Tilly JL. 
Prolongation of ovarian life span into advanced chronological age by BAX-deficiency. Nat Genet 1999; 21: 200-3.

18 Tilly JL, Tilly KI, Kenton ML, Johnson AL. Expression of members of the bcl-2 gene family in the immature rat ovary: equine chorionic gonadotropin-mediated inhibition of granulosa cell apoptosis is associated with decreased bax and constitutive bcl-2 and bcl-xlong messenger ribonucleic acid levels. Endocrinology 1995; 136: 230-6.

19 Zhai P, Zeng J, Tan N, Wang J, Huang L, She W. Effects of vitamin C on A549 cell proliferation, apoptosis and expressions of caspase, survivin. China J Lung Cancer 2010; 2: 89-94.

20 Arlt A, Vorndamm J, Breitenbroich M, Folsch UR, Kalthoff H, Schmidt $\mathrm{WE}$, et al. Inhibition of NF-KB sensitizes human pancreatic carcinoma cells to apoptosis induced by etoposide (VP16) or doxorubicin. Oncogene 2001; 20: 859-68.

21 Somerville L, Cory JG. Enhanced roscovitine-induced apoptosis is mediated by a caspase-3-like activity in deoxyadenosine-resistant mouse leukemia L1210 cells. Anticancer Res 2000; 20: 3347-55.

22 Manna SK, Aggarwal BB. Lipopolysaccharide inhibits TNF-induced apoptosis: role of nuclear factor-KB activation and reactive oxygen intermediates. J Immunol 1999; 162: 1510-8.

23 Wang S, Kotamraju S, Konorev E, Kalivendi S, Joseph J, Kalyanaraman B. Activation of nuclear factor-kappaB during doxorubicin-induced apoptosis in endothelial cells and myocytes is pro-apoptotic: the role of hydrogen peroxide. Biochem J 2002; 367: 729-40.

24 Hsu SC, Gavrilin MA, Lee HH, Wu CC, Han SH, Lai MZ. NF-kBdependent Fas ligand expression. Eur J Immunol 1999; 29: 294856.

25 Chan H, Bartos DP, Owen-Schaub LB. Activation-dependent transcriptional regulation of the human Fas promoter requires NF-KB p50/ p65 recruitment. Mol Cell Biol 1999; 19: 2098-108.

26 Qin ZH, Chen RW, Wang Y, Nakai M, Chuang DM, Chase TN. Nuclear factor $\mathrm{kB}$ nuclear translocation upregulates c-Myc and p53 expression during NMDA receptor-mediated apoptosis in rat striatum. J Neurosci 1999; 19: 4023-33.

27 Hettmann T, DiDonato J, Karin M, Leiden JM. An essential role for nuclear factor-kB in promoting double positive thymocyte apoptosis. J Exp Med 1999; 189: 145-58. 